\title{
Regulatory Region Deletion
}

National Cancer Institute

\section{Source}

National Cancer Institute. Regulatory Region Deletion. NCI Thesaurus. Code C148647.

A deletion mutation where the deleted sequence includes of a regulatory region for a gene. 